\title{
Artificial Immune System based PID Tuning for DC Servo Speed Control
}

\author{
Muna Hadi Saleh, PhD \\ Assist. Prof \\ Department of Electrical \\ Engineering/College of \\ Engineering/University of Baghdad
}

\author{
Saad Zaid Saad \\ Department of Electrical \\ Engineering/College of \\ Engineering/University of Baghdad
}

\begin{abstract}
In this paper, the application of the Artificial Immune System (AIS) algorithm for optimization problems is presented, DC Servo Motor (DCSM) speed control was taken as a case study. The PID controller was tuned using AIS algorithm by minimizing the Integral Time Absolute Error (ITAE). The results were compared with the results of the ZieglerNichols tuning method and it was obvious that the AIS gives better results. The AIS algorithm showed it has the ability to find the global optimum solution and it gave a response better than the response of the traditional tuning methods in terms of rise time, settling time, steady state error and overshoot.
\end{abstract}

\section{Keywords}

PID Controllers, Artificial Immune System (AIS), Ziegler Nichols (ZN), PID Optimization, DC Servo Motor (DCSM)

\section{INTRODUCTION}

DC Servo Motor (DCSM) has a significant speed control that made it widely used in the industry despite the maintenance costs of the induction motor are less than the maintenance of DCSM. As a result, many of modern PID tuning algorithms were used to give the best possible control. Artificial Immune System (AIS) is a new technique and it has wide applications not only in the optimization problems but also in data mining and pattern recognition problems [1]. The method inspired by the biological immune system of the human to solve real world problems. In nature, when antigens are threatened animal, a powerful immune response is advanced in order to save the organism, to do that a specific antibodies are created to defend the body and these antibodies go through hypermutation process to increase their ability to eliminate the antigens [2]. The general concept of the biological immune system is enough to inspire and develop the optimization algorithm, but if the problem that wanted to be solved is in another application such as pattern recognition, more details about the immune system are needed such as the immune cells types (B cells and T cells) [1].

\section{RELATED WORKS}

Thomas and Poongodi [3], presented a tuning method for DCSM position control by using Genetic Algorithm (GA) to select the PID gains. The results of the Genetic Algorithm based PID controller were compared with the results of Ziegler Nichols based PID controller. Carlos and Calvo [4], presented a new Artificial Immune System (AIS) optimization algorithm based on the clonal selection concept it is called Macro Artificial Immune System (Macro-AIS), the result of this method was compared with the result of the traditional AIS clonal selection algorithm. Bindu and Mini [5], presented a new method of tuning PID controller by using modified GA, it is called flexible GA tuning, and an optimal PID controller is tuned for DCSM.

\section{MATHEMATICAL MODEL OF THE DCSM}

The DCSM model is derived for armature controlled DCSM circuit as shown in Figure 1, that means the field current is constant [3].

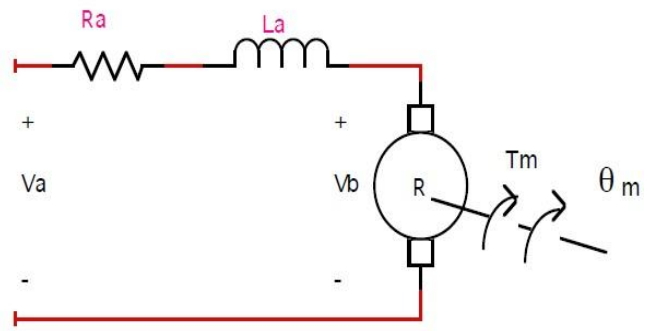

Figure 1: Armature controlled DC servo motor circuit

The electrical equations are:

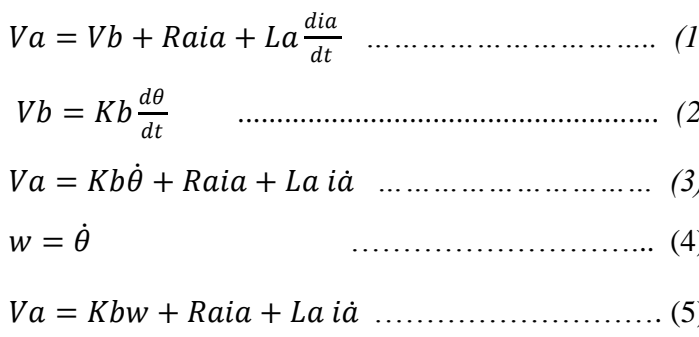

The mechanical equations are:

$T_{m}=K t i a$

$T_{m}=j o \ddot{\theta}+b o \dot{\theta}$

The physical parameters names and units are shown in Table $1[3]$.

Table 1. Physical parameters names and units

\begin{tabular}{|c|c|c|}
\hline Parameter & Name & Unit \\
\hline $\mathrm{Ra}$ & $\begin{array}{c}\text { Armature } \\
\text { resistance }\end{array}$ & Ohm \\
\hline $\mathrm{La}$ & $\begin{array}{c}\text { Armature } \\
\text { inductance }\end{array}$ & Henry \\
\hline ia & Armature current & Ampere \\
\hline $\mathrm{Va}$ & Armature voltage & Volt \\
\hline
\end{tabular}




\begin{tabular}{|c|c|c|}
\hline $\mathrm{Vb}$ & Back EMF & Volt \\
\hline $\mathrm{Kb}$ & $\begin{array}{c}\text { Back EMF } \\
\text { constant }\end{array}$ & $\frac{\text { volt }}{\mathrm{rad} / \mathrm{sec}}$ \\
\hline $\mathrm{Kt}$ & Torque constant & $\frac{\mathrm{N}-\mathrm{m}}{\text { ampere }}$ \\
\hline$T_{m}$ & Torque & $\mathrm{N}-\mathrm{m}$ \\
\hline$\theta(\mathrm{t})$ & $\begin{array}{c}\text { Angular } \\
\text { displacement }\end{array}$ & $\begin{array}{c}\text { Roment of } \\
\text { inertia }\end{array}$ \\
\hline Jo & $\begin{array}{c}\text { Frictional } \\
\text { constant of motor }\end{array}$ & $\frac{\mathrm{N}-\mathrm{mad} / \mathrm{sec}}{}$ \\
\hline bo & & \\
\hline
\end{tabular}

The physical parameters values for DCSM were selected as shown below [3]:

$\mathrm{Ra}=2.45, \mathrm{La}=0.035$, bo= $=0.5 * 10^{-2}, \mathrm{jo}=0.022$, and $\mathrm{Kt}=\mathrm{Kb}=1.2$.

By deriving the previous equations and substituting parameters values, the speed DCSM transfer function will be as shown below [3]:

$\frac{\theta(s)}{V a(s)}=\frac{1.2}{0.00077 s^{3}+0.539 s^{2}+1.44}$

\section{PID CONTROLLER TUNING}

PID controller is the most common controller used in the whole industries. PID controller is taken as a solution for many control problem because it has a simple model and due to the simplicity of the implementation [1].It is consist of three actions, proportional, derivative, and integral each action effect is controlled by its associated gain and respectively the gains are $\mathrm{Kp}, \mathrm{Ki}$, and $\mathrm{Kd}$. The aim of tuning the PID gains practically is to achieve a zero steady state error, low rising and settling times and low overshot [6]. The error minimization based tuning can achieve the entire mentioned objective. The traditional PID block diagram is shown in Figure 2.

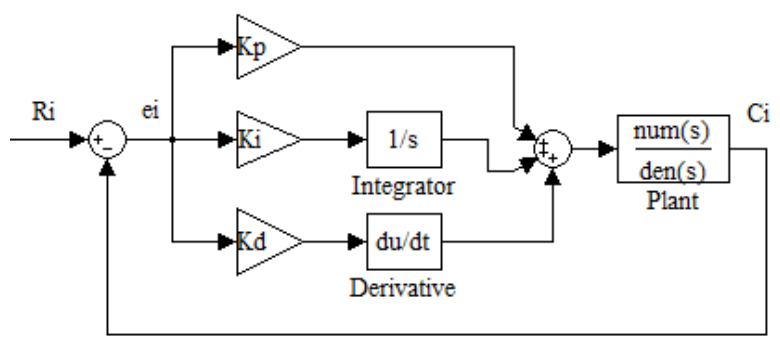

Figure 2. PID block diagram

The general PID equation for the figure showed above is:

$$
U_{i}(t)=e_{i}(t) * K p+\int e_{i}(t) * K i d t+\frac{d e_{i}}{d t} * K d
$$

Where $U_{i}$ is the input of the plant $\mathrm{G}(\mathrm{s}), e_{i}$ is the error and equal to $R_{i}-C_{i}$ Where $R_{i}, C_{i}$ are the input and the output of of the entire system respectively as shown in Figure (2), and $\mathrm{Kp}, \mathrm{Ki}$, and $\mathrm{Kd}$ are the gains of the PID controller.

\subsection{Ziegler-Nichols method}

Tuning by Ziegler-Nichols method is the most conventional PID tuning method and it was chosen as the main tuning method before finding the modern techniques [7]. There are two types of $\mathrm{ZN}$ tuning either closed loop tuning or open loop tuning, for DCSM speed control model open loop tuning method was used. The first step of the method is finding plant open loop response parameters, and the parameters are dead time $\left(D_{t}\right)$, time constant $\left(T_{C}\right)$, and the steady state gain $\left(K_{s t}\right)$. PID gains will be as shown in Table 2:

Table 2. ZN tuning table [6]

\begin{tabular}{|c|c|c|}
\hline $\mathrm{Kp}$ & $\mathrm{Ti}(\mathrm{sec})$ & $\mathrm{Td}(\mathrm{sec})$ \\
\hline$\frac{1.2 \mathrm{~T}_{\mathrm{C}}}{\mathrm{K}_{\mathrm{st}} \mathrm{D}_{\mathrm{t}}}$ & $2 \mathrm{D}_{\mathrm{t}}$ & $0.5 \mathrm{D}_{\mathrm{t}}$ \\
\hline
\end{tabular}

Where $\mathrm{Ki}=\mathrm{Kp} / \mathrm{Ti}, \mathrm{Kd}=\mathrm{Td} * \mathrm{Kp}$.

By applying the previous procedure the PID gains was calculated and the gains values are $\mathrm{Kp}=2.17, \mathrm{Ki}=67.05$, and $\mathrm{Kd}=0.017$.

\subsection{PID tuning by AIS algorithm}

The AIS algorithm was used to tune the PID three gains to get the best possible response depending on the minimization of the objective function that can take different types such as integral absolute error or integral squared absolute error. The strength of the optimization algorithms lies in finding the global solution for a problem has multiple optimum solutions (i.e. local optimum solutions) also in the accuracy of the solution and the time consumed to find it.

AIS has four different common techniques: Clonal Selection Algorithm, Negative Selection Algorithm, Immune Network Algorithms, and Dendritic Cell Algorithms [8]. In this paper, Clonal Selection and hyper mutation concepts were used and developed, in this method the main aim is to find the best antibodies with the best affinities to the solution. Best affinities mean best objective function value and that is lead to a maximization algorithm, The problem is minimizing the Integral Time Absolute Error (ITAE) so that the problem is adjusted to be a maximization of 1/ITAE. The optimization process steps are as shown below [8]:

1. Select the maximum number of generations, total number of antibodies ( $\mathrm{p}$ : population size), the number of antibodies selected to be cloned (n), the number of the worst antibodies selected to be replaced at the end of the generation $(\mathrm{k})$, and the number of antibodies selected to generate new random antibodies to save the diversity (d).

2. Apply clonal selection to the $\mathrm{n}$ antibodies: The clonal selection is applied directly proportional to the affinities of the antibodies, the antibody with high affinity generates high number of copies and the antibody with less affinity generates less copies and same thing to the other antibodies to generate $n^{\sim}$ of antibodies.

3. Apply hyper-mutation to the $n^{\sim}$ antibodies: Hypermutation is a normal mutation but with dynamic rate. The antibodies with high affinities will have a low mutation rate and vice versa.

4. Apply the elitism to check if the best antibody is not saved or not enhanced after the mutation, and brought it back to the population.

5. From the $n^{\sim}$ antibodies select the best different $n$ antibodies. 
6. From the remaining $\mathrm{p}-\mathrm{n}$ antibodies select worst $\mathrm{k}$ antibodies to replace them with $\mathrm{k}$ antibodies from $\mathrm{n}$.

7. From the remaining $\mathrm{p}-\mathrm{n}-\mathrm{k}$ select random $\mathrm{d}$ antibodies to generate new random antibodies to ensure the diversity [9].

8. Go to step 2 .

The stopping criterion used is: If there is no change in the error for many consecutive generations, the algorithm will be stopped. The block diagram in Figure 3 illustrates the above steps.

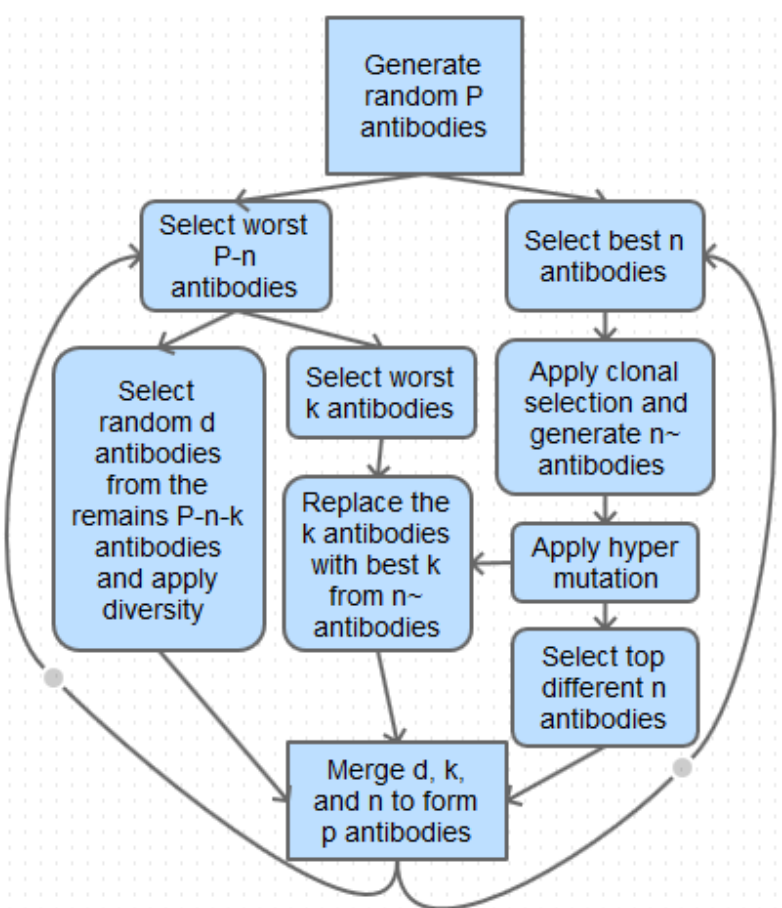

Figure 3 : Block diagram of the AIS clonal selection algorithm

\section{SIMULATION RESULTS}

The mentioned procedure was applied and population size of 100 antibodies, 10 diversity antibodies, 20 best antibodies to the clonal selection and 5 worst antibodies to replace it at the end of the generation were used. Real coded AIS was used, so that the antibody length is 3 variable. The important issue is the range or the maximum values of PID gains that give physically accepted response. Generally too big values are not preferred for more than one reason, the first reason is the power limitation of the controller output and the plant input. The second reason is to save the stability because if there is nonlinearity or uncertainty in the original model of the plant, too big gains maybe make the system in a very unstable scenario despite the simulation response is good. Three ranges were applied and they are (0-200), (0-100), and (0-50). Practically the third range is more preferable and reliable and gives a response close to the other ranges responses.

The AIS algorithms has an important feature, it needs less generations as compared with genetic algorithms to find the global optimal region in the search space. In this study case, due to small range of the 3 gains, the algorithm needs only one generation to reach the global region because best solution is cloned for 100 antibodies and each one of these 100 antibodies is go through hyper mutation process with very low mutation rate so that the best solution is enhanced. The objective function is the ITAE, but the integral is changed to summation of sampled version of the absolute error multiplied by the associated time with sample rate of $1000 \mathrm{sample/sec}$. The PID parameters for the three different ranges are shown in Table 3.

Table 3. AIS-PID gains

\begin{tabular}{|c|c|c|c|}
\hline Range of PID gains & Kp & Ki & Kd \\
\hline AIS(0 -200) & 7.48445 & 200 & 0.10675 \\
\hline AIS(0-100) & 3.64427 & 100 & 0.04993 \\
\hline AIS(0-50) & 1.678 & 50 & 0.01935 \\
\hline
\end{tabular}

The results shows that the AIS based gains are in the same range of the $\mathrm{ZN}$ based gains but with more accurate and less oscillatory response. The PID controller was applied to the DCSM to control the speed. The responses of the tuning methods are shown in Figure 4, Figure 5, Figure 6, and Figure 7. The last three figures are gathered in Figure 8 for comparison between them.

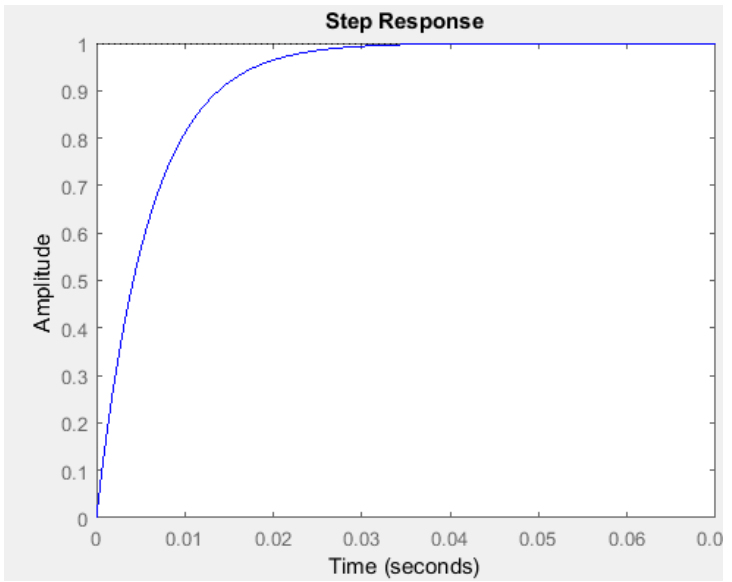

Figure 4 : Step response of AIS (0-200)-PID controller

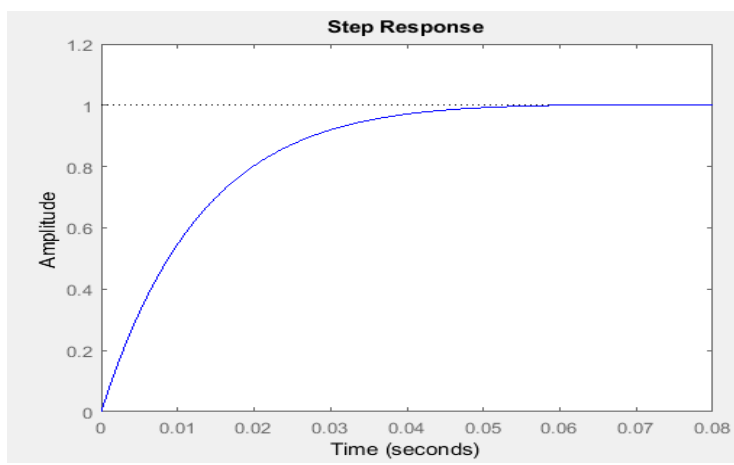

Figure 5 : Step response of AIS (0-100)-PID controller 


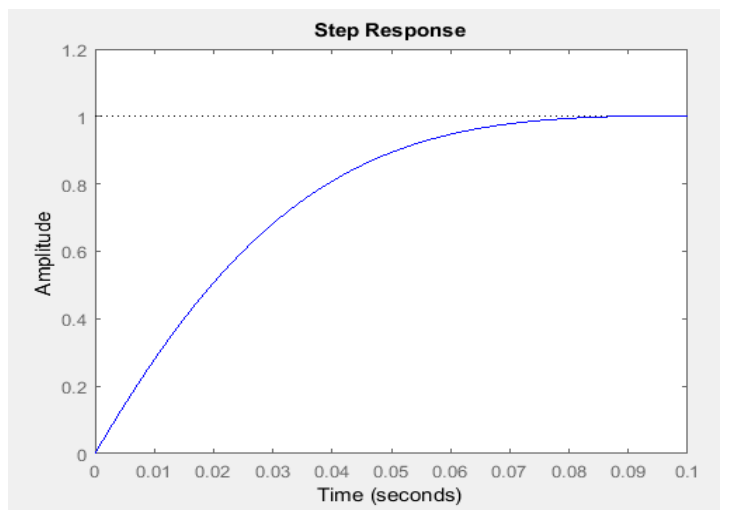

Figure 6 : Step response of AIS (0-50)-PID controller

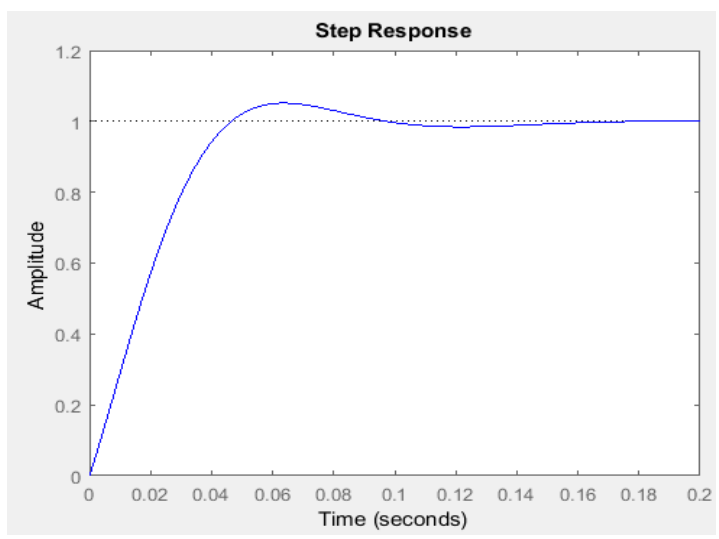

Figure 7 : Step response of ZN-PID controller

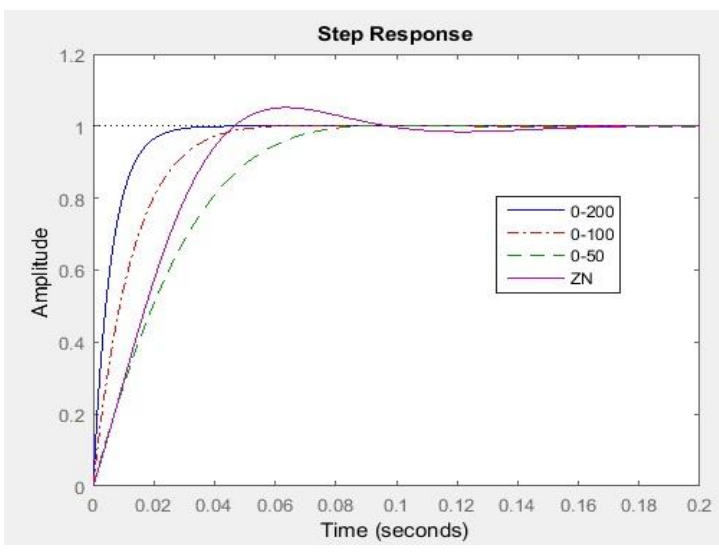

Figure 8 : Closed Loop Response of AIS-PID and ZN-PID Controllers

Performance index (PI) or step response characteristics in terms of overshoot, settling time, rise time, and steady state error with the values of ITAE are illustrated in Table 4.

Table 4.Step response parameters

\begin{tabular}{|c|c|c|c|c|}
\hline Method & ZN & AIS & AIS & AIS \\
\hline $\begin{array}{c}\text { Rise } \\
\text { time(sec.) }\end{array}$ & 0.033 & 0.0476 & 0.0263 & 0.0132 \\
\hline Mp \% & 5.1713 & 0.2405 & 0.1955 & 0 \\
\hline
\end{tabular}

\begin{tabular}{|c|c|c|c|c|}
\hline $\begin{array}{c}\text { Settling } \\
\text { time(sec.) }\end{array}$ & 0.0855 & 0.0707 & 0.0431 & 0.0234 \\
\hline $\begin{array}{c}\text { Steady state } \\
\text { error \% }\end{array}$ & 0.0182 & 0.2398 & 0.1909 & 0.0014 \\
\hline ITAE & 0.4556 & 0.49508 & 0.14079 & 0.036335 \\
\hline
\end{tabular}

Where $\mathrm{Mp}$ is the peak overshoot and P.I is the performance index.

\section{CONCLUSION}

The previous results show that the controller that tuned using AIS algorithm gives a very small overshoot approximately zero but in $\mathrm{ZN}$ based controller the response has an overshoot of $5.17 \%$. The important point is Ki maximum value plays a major role in determining the response shape and mathematically it can takes a values greater than 200 but to make the system more realizable the range of the gains restricted in three different ranges even the range that is below $\mathrm{ZN}$ tuned $\mathrm{Ki}$ value gives a good response. As an overall, the compromise between the performance indices and the physical acceptance shows that the AIS (0-50) tuned PID gives the best response.

The future scope of this work is using AIS both common algorithms (clonal selection and artificial immune network) in more complicated problem of learning and optimizing seismic attributes for subsurface reservoir studies, and compare the results of both algorithms to infer which algorithm is better.

\section{REFERENCES}

[1] Edmund K. Burke . Graham Kendall, "Artificial Immune System," in Search Methodologies,2nd ed. New York City: springer, sec. 7.2.4, pp. 191, 2014.

[2] J.R. Al-Enezi, M.F. Abbod \& S. Alsharhan,“ Artificial Immune Systems - Models, Algorithms And Applications," in International Journal of Research and Reviews in Applied Sciences, IJRRAS, 2010.

[3] Neenu Thomas and Dr. P. Poongodi, "Position Control of DC Motor Using Genetic Algorithm Based PID Controller," in World Congress on Engineering., London., WCE, 2009.

[4] Juan Carlos et al., "A Micro Artificial Immune System" in Polibits, Mexico City, Mexico, 2011.

[5] Bindu R. and Mini K., "Tuning of PID Controller for DC Servo Motor using Genetic Algorithm" in International Journal of Emerging Technology and Advanced Engineering, New Delhi, India, IJETAE, 2012.

[6] Rames C. Panda, "PID Controller Design for Specified Performance," in Introduction To PID Controllers Theory, Tuning And Application To Frontier Areas, 1st ed. :In Tech, sec. 1, pp. 4, 2012.

[7] katsuhiko ogata, "PID Controllers and Modified PID Controllers," in Modern Control Engineering, 5th ed.: New Jersey,Prentice Hall, sec. 8.2, pp. 568, 2010.

[8] Raymond Chiong, "Social-Natural Intelligence," in Nature-Inspired Algorithms for Optimisation, 1st ed. New York City: springer, sec. IV, pp. 389, 2009.

[9] Maoguo Gong et al., "Orthogonal Immune Algorithm with Diversity-based Selection for Numerical Optimization," in Association for Computing Machinery., New York City., ACM, 2009. 